\title{
Heavy-flavour production in heavy-ion collisions at RHIC and at the LHC
}

\section{Diego Stocco*}

Subatech - Laboratoire de Physique Subatomique et des Technologies Associées, Nantes, France

E-mail: stoccolsubatech.in2p3. fr

Heavy flavour measurements have a key role in the investigation of the properties of the hot and dense state of strongly interacting matter which is produced in ultra-relativistic nucleus-nucleus collisions. Heavy quarks are produced in the hard scattering processes occurring at the initial stages of the collision, and act as self-generated probes experiencing the full evolution of the medium. The measurement of open charm and beauty allows one to probe the mechanisms of heavy-quark propagation, energy loss and hadronisation in the medium.

A systematic study of heavy flavour production in nucleus-nucleus collisions was first carried out at the Relativistic Heavy Ion Collider at the Brookhaven National Laboratory, and then continued both at RHIC and at the Large Hadron Collider at CERN, with a significative increase in the centre of mass energy. The nuclear modification factor and azimuthal anisotropy of heavy flavours in several decay channels have been measured in a wide kinematic range. A selection of the results will be presented in these proceedings and the implication for the properties of the medium will be discussed.

The European Physical Society Conference on High Energy Physics 22-29 July 2015

Vienna, Austria

\footnotetext{
* Speaker.
} 


\section{Introduction}

Heavy-flavour hadrons (with a charm or beauty content), are effective probes of the QuarkGluon Plasma (QGP) which is formed in ultra-relativistic heavy-ion collisions. Heavy quark pairs are produced in the hard scattering processes at the initial stages of the collision, with a minimum virtuality of the order of the pair mass $\left(2 m_{Q}\right)$. This implies a formation time of $\tau=1 /\left(2 m_{c}\right) \sim$ $0.07 \mathrm{fm} / c$ for charm and $1 /\left(2 m_{b}\right) \sim 0.02 \mathrm{fm} / c$ for beauty, which is shorter than the formation time of the plasma $(0.1-1 \mathrm{fm} / c)$. Heavy quarks behave hence like initially-produced probes which are exposed to the medium evolution. Unlike light quarks and gluons, the charm and beauty quarks preserve their flavour and mass identity while traversing the medium, and can hence be tagged via the measurement of heavy-flavour hadrons.

Several approaches have been developed to describe the interaction of heavy quarks with the surrounding plasma [1]. In a perturbative treatment, the partons lose part of their energy via gluon radiation and elastic scattering. The energy loss is expected to be larger for partons with a larger colour charge (gluons), and smaller for heavy quarks with a moderate energy-over-mass ratio, since the small-angle gluon radiation is reduced (dead-cone effect [2]). This charge and mass dependence can be investigated via a comparative study of the light hadrons (mostly originated from gluons), D mesons and B mesons. However, other mechanisms such as in medium formation and dissociation have to be taken into account in the comparison of the hadronic observables.

At low transverse momenta, the interaction with the medium could be large enough for the heavy quarks to participate in the collective expansion of the medium, possibly reaching thermal equilibrium with it. In this $p_{\mathrm{T}}$ range, the hadronization might predominantly occur inside the medium via the coalescence mechanism rather than via fragmentation in the vacuum.

To answer these open questions, systematic measurements have been performed both at the Relativistic Heavy Ion Collider (RHIC) and at the Large Hadron Collider (LHC), at significantly larger energies. A selection of the results will be presented in the following.

\section{Heavy-flavour measurements at RHIC and at the LHC}

Heavy-flavours are reconstructed with various techniques exploiting the long lifetime of the charm $(c \tau \sim 100-300 \mu \mathrm{m})$ and beauty $(c \tau \sim 500 \mu \mathrm{m})$ quarks. Electrons from heavy-flavour decays are measured at mid-rapidity by the PHENIX [3] and STAR [4] experiments at RHIC and by the ALICE [5] experiment at the LHC. In the PHENIX experiment, the electron identification is based on the Ring Imaging Čherenkov in conjunction with a high-granularity Electro-Magnetic Calorimeter (EMCal). The background contribution, mainly consisting of Dalitz decays of light neutral mesons and photon conversions in the material, is parameterised with a Monte Carlo cocktail and subtracted from the inclusive spectrum. A complementary method exploiting an additional photon converter is used as well for background subtraction. The STAR experiment identifies electrons using the specific energy loss in the Time Projection Chamber (TPC), together with the TimeOf-Flight (TOF) information for $p_{\mathrm{T}}<1.5 \mathrm{GeV} / c$ or with the EMCal at larger $p_{\mathrm{T}}$. The background contribution is subtracted in a statistical way through the measurement of the $e^{+} e^{-}$invariant mass. In the ALICE experiment, electrons are identified using the TPC, TOF, EMCal and the Transition 
Radiation Detector. The background subtraction is performed with both the cocktail and invariant mass methods.

Muons from heavy-flavour decays are measured by the PHENIX experiment at RHIC and by the ALICE and ATLAS [6] experiments at the LHC. In the PHENIX experiment, muons are reconstructed in two muon spectrometers covering the pseudo-rapidity interval $-2.2<\eta<-1.2$ and $1.2<\eta<2.4$. The irreducible hadronic background is described by a data-driven Monte Carlo cocktail and subtracted. An analogous method for signal extraction is adopted by the ALICE experiment, which measures muons in the pseudo-rapidity interval $-4<\eta<-2.5$. In the ATLAS experiment, muons are reconstructed at mid-rapidity $(|\eta|<1.05)$ by matching the tracks in the Inner silicon Detector with the ones in the Muon Spectrometer, surrounding the electromagnetic and hadronic calorimeter. The background sources are subtracted though a Monte Carlo template fit of discriminant selection variables.

Charmed mesons are reconstructed in the STAR and ALICE experiments through the invariant mass analysis of the full hadronic decay topologies $\left(\mathrm{D}^{0} \rightarrow K^{-} \pi^{+}, \mathrm{D}^{+} \rightarrow K^{-} \pi^{+} \pi^{+}, \mathrm{D}^{*+} \rightarrow \mathrm{D}^{0} \pi^{+}\right.$, $\mathrm{D}_{\mathrm{s}}^{+} \rightarrow K^{+} K^{-} \pi^{+}$). In both experiments, pions are identified by combining the information of the specific energy loss in the TPC and of the Time-of-Flight. The spatial resolution of the inner tracker of ALICE allows, in addition, to reconstruct the secondary vertices of the D meson decays.

The semi-inclusive beauty production can be studied both in ALICE and CMS [7] through the measurement of displaced $\mathrm{J} / \psi$ from B decays, which is based on the simultaneous fit of the $e^{+} e^{-}$ (ALICE) or $\mu^{+} \mu^{-}$(CMS) invariant mass and the pseudo-proper decay length. In addition, the $\mathrm{CMS}$ experiment measured $\mathrm{b}$-tagged jets for the first time in $\mathrm{Pb}-\mathrm{Pb}$ collisions by direct reconstruction of the displaced vertex associated to the jet.

\section{Heavy-ion results}

The nuclear modification factor $\left(\mathrm{R}_{\mathrm{AA}}\right)$ is a sensitive observable to study the interaction of hard partons with the medium formed in heavy-ion collisions. The $\mathrm{R}_{\mathrm{AA}}$ is defined as the ratio of the heavy-flavour yields measured in heavy-ion collisions $\left(\mathrm{d} N_{A A} / \mathrm{d} p_{\mathrm{T}}\right)$ and in pp collisions $\left(\mathrm{d} N_{p p} / \mathrm{d} p_{\mathrm{T}}\right)$ scaled by the average number of nucleon-nucleon collisions $\left(\left\langle N_{\text {coll }}\right\rangle\right)$ :

$$
\mathrm{R}_{\mathrm{AA}}=\frac{1}{\left\langle N_{\mathrm{coll}}\right\rangle} \frac{\mathrm{d} N_{A A} / \mathrm{d} p_{\mathrm{T}}}{\mathrm{d} N_{p p} / \mathrm{d} p_{\mathrm{T}}}
$$

In the absence of nuclear or medium effects, the heavy-flavour production is expected to scale as the number of binary nucleon-nucleon collisions (binary scaling), thus resulting in a $\mathrm{R}_{\mathrm{AA}}$ of 1 . The binary scaling is broken down by initial state effects (such as the nuclear modification of the parton distribution functions, gluon saturation at small Bjorken- $x$ values or $k_{t}$ broadening), or by the interaction with the medium. In particular, the in-medium energy loss leads to a softening of the heavy-flavour momentum spectrum, which results in a $\mathrm{R}_{\mathrm{AA}}<1$ at high transverse momenta. The colour charge and mass dependence of the energy loss should give rise to a hierarchy in the nuclear modification factors of light hadrons, charmed hadrons and beauty hadrons, with $\mathrm{R}_{\mathrm{AA}}^{\mathrm{h}}<$ $\mathrm{R}_{\mathrm{AA}}^{\mathrm{D}}<\mathrm{R}_{\mathrm{AA}}^{\mathrm{B}}$.

The PHENIX and STAR experiments measured the nuclear modification factor of electrons from heavy-flavour decays in $\mathrm{Au}-\mathrm{Au}$ collisions at $\sqrt{s_{\mathrm{NN}}}=200 \mathrm{GeV}[8,9]$. A strong suppression of 
a factor up to $3-5$ relative to pp collisions is observed at $p_{\mathrm{T}}>5 \mathrm{GeV} / c$, compatible with the one of pions in a similar transverse momentum range [10]. The strong effect is not observed in $\mathrm{d}-\mathrm{Au}$ collisions at the same energy (Figure 1), and should hence be attributed to the interaction with the medium formed in $\mathrm{Au}-\mathrm{Au}$ collisions. The PHENIX experiment also measured the production of

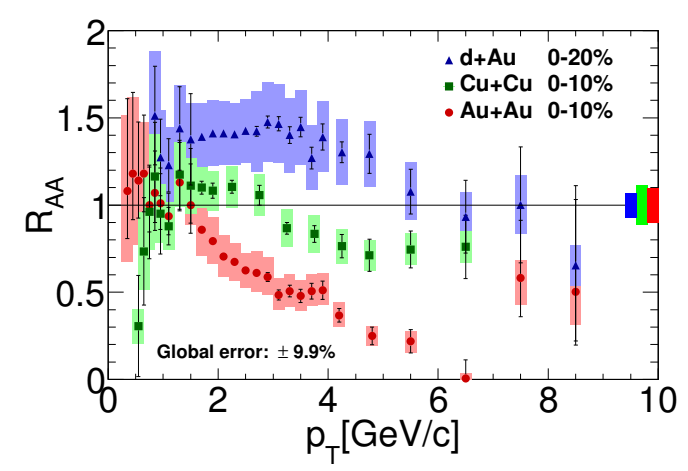

Figure 1: Transverse momentum dependence of electrons from heavy-flavour decays measured in d-Au, $\mathrm{Cu}-\mathrm{Cu}$ and $\mathrm{Au}-\mathrm{Au}$ collisions at $\sqrt{s_{\mathrm{NN}}}=200 \mathrm{GeV}$ by the PHENIX experiment [8].

heavy-flavour decay muons at forward rapidity in $\mathrm{Cu}-\mathrm{Cu}$ collisions at $200 \mathrm{GeV}$ [11]: a stronger suppression is observed compared to the electron measurements at mid-rapidity.

At the LHC, the heavy-flavour production is measured in the semi-electronic decay channel at mid-rapidity by the ALICE experiment [12] and in the semi-muonic decay channel at forward and mid-rapidity by the ALICE [13] and ATLAS [14] experiment, respectively. A large reduction of the yields is observed as well, although the value of $\mathrm{R}_{\mathrm{AA}}$ is similar at forward and mid rapidity, thus indicating a weak dependence of this variable in the region $|y|<4$.

The charm production was measured in the $\mathrm{D}^{0} \rightarrow K^{-} \pi^{+}$decay channel by the STAR experiment in $\mathrm{Au}-\mathrm{Au}$ collisions at $\sqrt{s_{\mathrm{NN}}}=200 \mathrm{GeV}$ [15]. The $\mathrm{R}_{\mathrm{AA}}$ as a function of $p_{\mathrm{T}}$ is shown in the left panel of Figure 2. For $p_{\mathrm{T}}>3 \mathrm{GeV} / c$, values of $0.3-0.5$ are reached, compatible with the pion results [16]. For $p_{\mathrm{T}}$ of $1-2 \mathrm{GeV} / c$, the $\mathrm{R}_{\mathrm{AA}}$ exhibits an enhancement above unity, which can be qualitatively described by model calculations including collisional interactions in an expanding medium, and a contribution of hadronisation via in-medium quark recombination [1]. The ALICE experiment measured $\mathrm{D}^{0}, \mathrm{D}^{+}$and $\mathrm{D}^{*+}$ in $\mathrm{Pb}-\mathrm{Pb}$ collisions at $\sqrt{s_{\mathrm{NN}}}=2.76 \mathrm{TeV}[17,18]$. The average $\mathrm{R}_{\mathrm{AA}}$ of $\mathrm{D}$ mesons is shown in the right panel of Figure 2: a strong suppression is observed (factor of 4-5) at high- $p_{\mathrm{T}}(5-30 \mathrm{GeV} / c)$. At variance with RHIC, at the LHC the $\mathrm{R}_{\mathrm{AA}}$ is smaller than unity down to $p_{\mathrm{T}}=1 \mathrm{GeV} / c$. It is worth noting, however, that the $p_{\mathrm{T}}$ distribution is harder in the latter case and the gluon shadowing is more pronounced. The ALICE experiment also measured the $\mathrm{D}_{\mathrm{s}}$ for the first time in $\mathrm{Pb}-\mathrm{Pb}$ collision. In a strangeness-rich plasma, the $\mathrm{D}_{\mathrm{s}}$ meson production is expected to be enhanced compared to pp collisions, if the coalescence is the main hadronisation mechanism. However, the $\mathrm{D}_{\mathrm{s}}$ measurement is still affected by large uncertainties: more data are needed in order to draw a firm conclusion.

The beauty frontier in heavy-ion collisions was first explored by the CMS experiment, with the measurement of b-tagged jets [19] and of $\mathrm{J} / \psi$ from $\mathrm{B}$ meson decays in $\mathrm{Pb}-\mathrm{Pb}$ collisions at $\sqrt{s_{\mathrm{NN}}}=$ $2.76 \mathrm{TeV}$ [20]. The centrality dependence of the $\mathrm{R}_{\mathrm{AA}}$ of $\mathrm{b}$-jets is shown in the left panel of Figure 3: the suppression increases from the most peripheral to the most central collisions, reaching a factor 

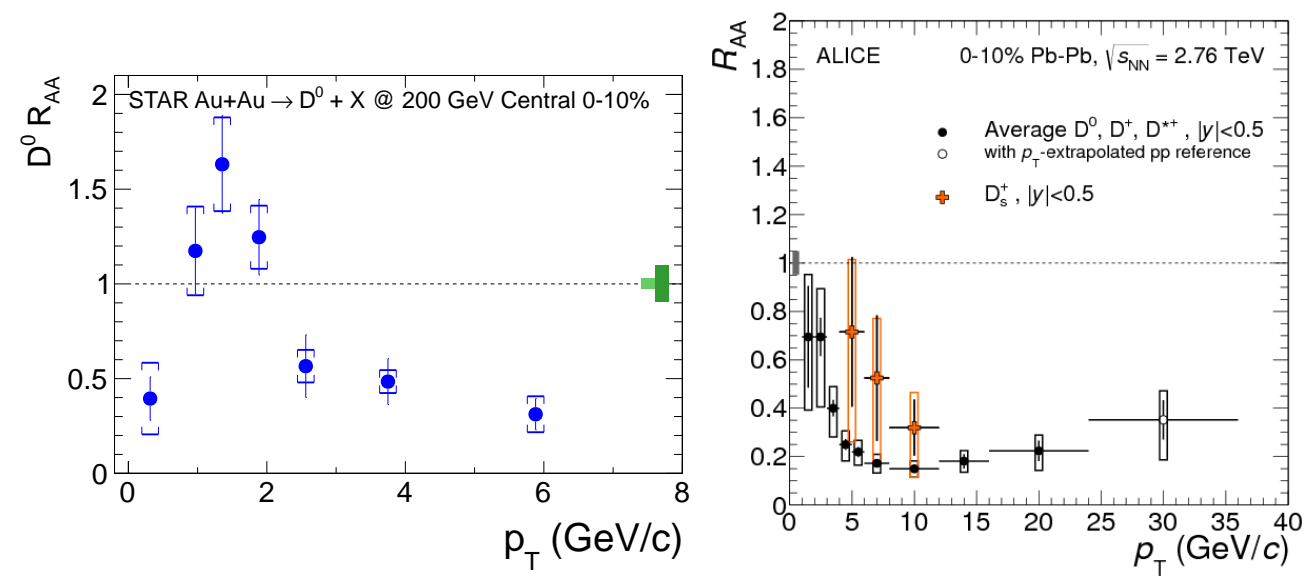

Figure 2: Left panel: nuclear modification factor of $\mathrm{D}^{0}$ mesons in central $\mathrm{Au}-\mathrm{Au}$ collisions at $\sqrt{s_{\mathrm{NN}}}=$ $200 \mathrm{GeV}$ [15]. Right panel: nuclear modification factor of the average of prompt D mesons (black circles) and $\mathrm{D}_{\mathrm{s}}$ mesons (open orange crosses) in central $\mathrm{Pb}-\mathrm{Pb}$ collisions at $\sqrt{s_{\mathrm{NN}}}=2.76 \mathrm{TeV}$ [18].

of about 2.5. The measured $\mathrm{R}_{\mathrm{AA}}$ is found to be compatible with the one of inclusive jets [21], which is dominated by gluon jets at large $p_{\mathrm{T}}$. While a quark mass effect might not play a role at such values of $p_{\mathrm{T}}$, the colour charge dependence of energy loss should manifest itself as a difference in the $\mathrm{b}$-jet and inclusive jets $\mathrm{R}_{\mathrm{AA}}$. It is worth noting, however, that at high $p_{\mathrm{T}} \mathrm{b}$-jets mainly originate from gluon splitting, which means that a significant fraction of the in-medium path length is traversed by the parent gluon.
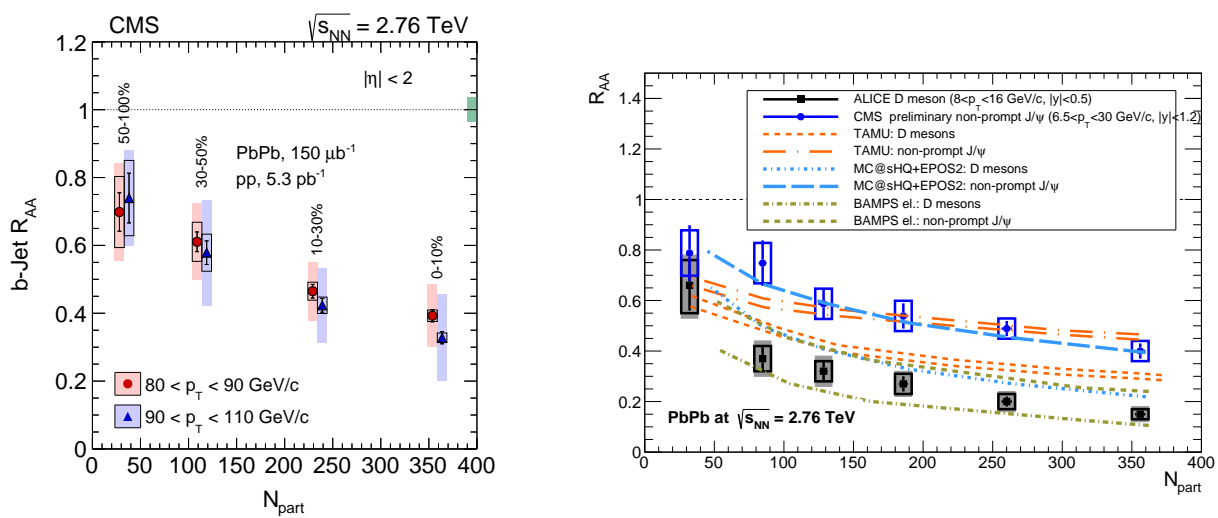

Figure 3: Left panel: nuclear modification factor of b-jets as a function of the number of participants in $\mathrm{Pb}-\mathrm{Pb}$ collisions at $\sqrt{s_{\mathrm{NN}}}=2.76 \mathrm{TeV}$ [19]. Right panel: $\mathrm{R}_{\mathrm{AA}}$ of secondary $\mathrm{J} / \psi$ from $\mathrm{B}$ meson decay in $\mathrm{Pb}-\mathrm{Pb}$ collisions at $\sqrt{s_{\mathrm{NN}}}=2.76 \mathrm{TeV}$ [20], compared to the $\mathrm{R}_{\mathrm{AA}}$ of prompt $\mathrm{D}$ meson [22]. Theoretical predictions are also shown (see [1] and references therein).

The centrality dependence of the $\mathrm{R}_{\mathrm{AA}}$ of $\mathrm{J} / \psi$ from $\mathrm{B}$ meson decays is shown in the right panel of Figure 3. The results are compared with the $\mathrm{R}_{\mathrm{AA}}$ of $\mathrm{D}$ mesons, measured in a $p_{\mathrm{T}}$ range chosen so that the average $\mathrm{D}$ meson $p_{\mathrm{T}}$ is similar to the one probed by the $\mathrm{B}$ meson. The $\mathrm{R}_{\mathrm{AA}}$ of nonprompt $\mathrm{J} / \psi$ is found to be larger than the one of $\mathrm{D}$ mesons. The difference is explained by models implementing a mass-dependent energy loss [1]. 
The information provided by the $\mathrm{R}_{\mathrm{AA}}$ can be complemented by the study of the azimuthal anisotropy of heavy-flavour production with respect to the reaction plane. The latter is defined by the beam axis and the impact parameter vector of the two colliding nuclei. If the medium is characterised by a large collectivity, possibly leading to the thermalisation of heavy flavours, the almond-shaped initial geometry of a non-central heavy-ion collision is transformed in an anisotropy in the momentum space. The anisotropy can be experimentally quantified by means of the second order coefficient $\left(v_{2}\right)$ of the Fourier harmonic expansion of the azimuthal distribution, the so called "elliptic flow".

The PHENIX and STAR experiment measured the elliptic flow of electrons from heavy-flavour decays in $\mathrm{Au}-\mathrm{Au}$ collisions at different energies. The results at $\sqrt{s_{\mathrm{NN}}}=200 \mathrm{GeV}[23,24]$ show a non-null $v_{2}$, with a maximum at $p_{\mathrm{T}} \sim 1.5 \mathrm{GeV} / c$ (see left panel of Figure 4 for PHENIX results). At larger $p_{\mathrm{T}}$, the data suggest a decreasing trend, although the errors are too large to draw a firm conclusion. At lower energies $\left(\sqrt{s_{\mathrm{NN}}}=64.2 \mathrm{GeV}\right.$ [25] and $39 \mathrm{GeV}$ [24]), the central value of the $v_{2}$ is significantly smaller than the one measured at $\sqrt{s_{\mathrm{NN}}}=200 \mathrm{GeV}$, although the statistical and systematic uncertainties are sizeable.
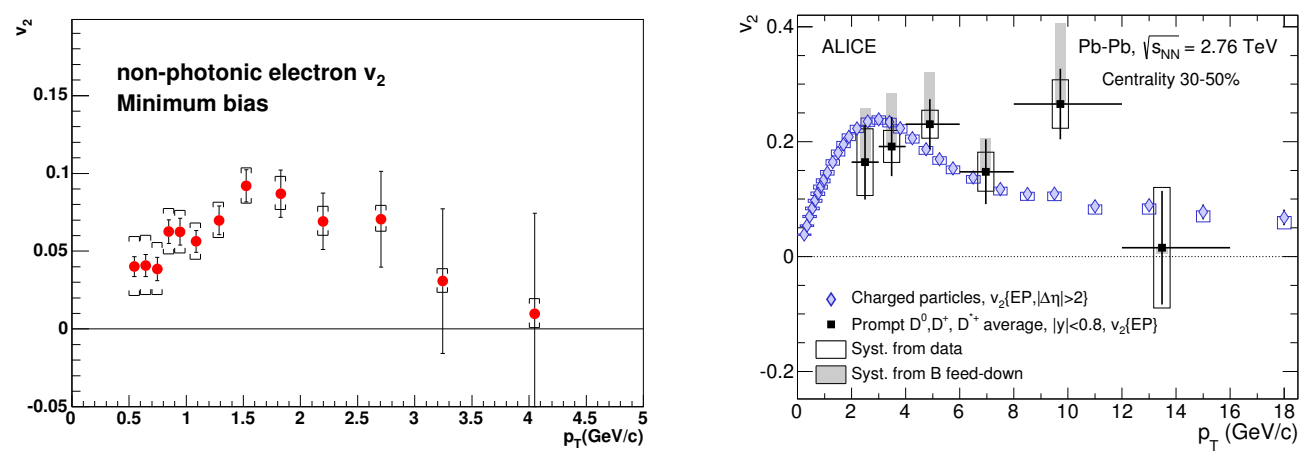

Figure 4: Left panel: elliptic flow of electrons from heavy-flavour decays in Au-Au collisions at $\sqrt{s_{\mathrm{NN}}}=$ $200 \mathrm{GeV}$ [23]. Right panel: elliptic flow of the average of prompt $\mathrm{D}$ mesons in $\mathrm{Pb}-\mathrm{Pb}$ collisions at $\sqrt{s_{\mathrm{NN}}}$ $=2.76 \mathrm{TeV}$ [27] (black squares). The analogous measurement for charged particles is shown as well (blue diamonds).

At LHC energies, the ALICE experiment measured a non-null $v_{2}$ of heavy-flavour decay electrons at mid-rapidity and muons at forward rapidity [26]. The results are comparable with each other and with the ones of RHIC top energy. The ALICE experiment has also carried out the direct measurement of prompt D meson $v_{2}$. The results for the 30-50\% centrality class are shown in the right panel of Figure 4: the $v_{2}$ is found to be larger than 0 in the range $2<p_{\mathrm{T}}<6 \mathrm{GeV} / c$, with a $5.7 \sigma$ significance. The measured $v_{2}$ is compatible with the one of charged particles, although the uncertainties still prevent to draw a firm conclusion.

The observed anisotropy at both RHIC and LHC are described by several models that include quark transport in the medium, in particular by the ones describing a collisional interaction within a fluid expanding dynamic medium, as well as hadronisation via recombination [1]. On the other hand, the models that include radiative and collisional energy loss provide a good description of the $\mathrm{D}$ meson $\mathrm{R}_{\mathrm{AA}}$, but tend to under-predict the measured value of $v_{2}$ [1]. 
The upgrades in the silicon detector at both RHIC and LHC and the increase in heavy-ion luminosity are expected to open up a new era in heavy-flavour studies, both by increasing the precision of the described observables and by allowing qualitatively new measurements, such as the heavy-flavour baryon production or the flow of B mesons.

\section{Conclusions}

A selection of the heavy-flavour measurements in heavy-ion collisions at RHIC and at the LHC has been presented and discussed. The nuclear modification factor of heavy flavours was measured in different decay channels and in a large kinematic range. The results show a strong reduction of heavy-flavour yields at large $p_{\mathrm{T}}$ compared to the measurements in proton-proton collisions. The suppression is not observed in proton-nucleus (deuteron-nucleus) collisions and can hence be attributed to the in-medium energy loss. While there is still no experimental evidence of the charge dependence of the energy loss, the observation of a stronger suppression of $\mathrm{D}$ meson production compared to the one of $\mathrm{J} / \psi$ from B meson decays is consistent with the expectation of a lower in-medium energy loss of charm quark than beauty quark. The measurement of the elliptic flow of heavy flavours brings additional information. At low $p_{\mathrm{T}}$, a positive $v_{2}$ is observed in noncentral collisions both at RHIC and at the LHC, similar to the one of light hadrons within large uncertainties. The measurements indicate that the interaction with the medium constituents transfer information about the azimuthal anisotropy of the system to the heavy quark.

Several theoretical models implementing in-medium energy loss and/or hadronisation can describe the data, at least qualitatively [1]. However, further development is needed in order to connect the data with the fundamental properties of the QGP and of the theory of the strong interaction. From the experimental point of view, the upgrade of the silicon detectors at both RHIC and LHC as well as the increase in the luminosity is expected to improve the precision of the current measurements and to allow qualitatively new measurements. This will be essential to further improve our understanding of the hot nuclear medium with hard probes.

\section{References}

[1] A. Andronic et al., arXiv:1506.03981 [nucl-ex].

[2] Y. L. Dokshitzer and D. E. Kharzeev, Phys. Lett. B 519 (2001) 199 [hep-ph/0106202].

[3] K. Adcox et al. [PHENIX Collaboration], Nucl. Instrum. Meth. A 499 (2003) 469.

[4] K. H. Ackermann et al. [STAR Collaboration], Nucl. Instrum. Meth. A 499 (2003) 624.

[5] K. Aamodt et al. [ALICE Collaboration], JINST 3 (2008) S08002.

[6] G. Aad et al. [ATLAS Collaboration], JINST 3 (2008) S08003.

[7] S. Chatrchyan et al. [CMS Collaboration], JINST 3 (2008) S08004.

[8] A. Adare et al. [PHENIX Collaboration], Phys. Rev. C 90 (2014) 3, 034903 [arXiv:1310.8286 [nucl-ex]].

[9] B. I. Abelev et al. [STAR Collaboration], Phys. Rev. Lett. 98 (2007) 192301 [Phys. Rev. Lett. 106 (2011) 159902] [nucl-ex/0607012]. 
[10] S. S. Adler et al. [PHENIX Collaboration], Phys. Rev. Lett. 91 (2003) 072301 [nucl-ex/0304022].

[11] A. Adare et al. [PHENIX Collaboration], Phys. Rev. C 86 (2012) 024909 [arXiv:1204.0754 [nucl-ex]].

[12] S. Sakai [ALICE Collaboration], Nucl. Phys. A $904-905$ (2013) 661c.

[13] B. Abelev et al. [ALICE Collaboration], Phys. Rev. Lett. 109 (2012) 112301 [arXiv:1205.6443 [hep-ex]].

[14] [ATLAS Collaboration], ATLAS-CONF-2012-050.

[15] L. Adamczyk et al. [STAR Collaboration], Phys. Rev. Lett. 113 (2014) 14, 142301 [arXiv:1404.6185 [nucl-ex]].

[16] G. Agakishiev et al. [STAR Collaboration], Phys. Rev. Lett. 108 (2012) 072302 [arXiv:1110.0579 [nucl-ex]].

[17] J. Adam et al. [ALICE Collaboration], arXiv:1509.06888 [nucl-ex].

[18] J. Adam et al. [ALICE Collaboration], arXiv:1509.07287 [nucl-ex].

[19] S. Chatrchyan et al. [CMS Collaboration], Phys. Rev. Lett. 113 (2014) 13, 132301 [Phys. Rev. Lett. 115 (2015) 2, 029903] [arXiv:1312.4198 [nucl-ex]].

[20] S. Chatrchyan et al. [CMS Collaboration], CMS-PAS-HIN-12-014.

[21] S. Chatrchyan et al. [CMS Collaboration], CMS-PAS-HIN-12-004.

[22] J. Adam et al. [ALICE Collaboration], arXiv:1506.06604 [nucl-ex].

[23] A. Adare et al. [PHENIX Collaboration], Phys. Rev. Lett. 98 (2007) 172301 [nucl-ex/0611018].

[24] L. Adamczyk et al. [STAR Collaboration], arXiv:1405.6348 [hep-ex].

[25] A. Adare et al. [PHENIX Collaboration], Phys. Rev. C 91 (2015) 4, 044907 [arXiv:1405.3301 [nucl-ex]].

[26] J. Adam et al. [ALICE Collaboration], arXiv:1507.03134 [nucl-ex].

[27] B. Abelev et al. [ALICE Collaboration], Phys. Rev. Lett. 111 (2013) 102301 [arXiv:1305.2707 [nucl-ex]]. 